\title{
Retraction Note to: The distribution of the maximum of a variance gamma process and path-dependent option pricing
}

\author{
Roman V. Ivanov 1
}

Published online: 29 March 2016

(C) Springer-Verlag Berlin Heidelberg 2016

\section{Retraction Note to: Finance Stoch (2015) 19: 979-993 DOI 10.1007/s00780-015-0277-8}

The article "The distribution of the maximum of a variance gamma process and path-dependent option pricing" published in Volume 19/4, pages 979-993, DOI 10.1007/s00780-015-0277-8 has been retracted by agreement between the author Roman V. Ivanov and the journal's Editors Martin Schweizer and Chris Rogers. The retraction has been agreed because the paper contains a fundamental error which invalidates the results of the paper. The paper studies a variance gamma process as a time change of Brownian motion, but in Sect. 5 the proof of Theorem 2.1 incorrectly assumes that the pathwise maximum of the time-changed Brownian motion is the time-change of the maximum of the Brownian motion. We are grateful to Alexey Kuznetsov for detecting this error and drawing it to our attention.

The online version of the original article can be found under doi:10.1007/s00780-015-0277-8.

R.V. Ivanov

roivanov@yahoo.com

1 Laboratory of Control under Incomplete Information, Trapeznikov Institute of Control Sciences of RAS, Profsoyuznaya 65, 117997 Moscow, Russian Federation 